\title{
L'instruction en français du Chinois Kan Gao sous la Restauration à Paris
}

Laurent Puren

\section{Q OpenEdition \\ 1 Journals}

Édition électronique

URL : https://journals.openedition.org/dhfles/3459

DOI : $10.4000 /$ dhfles.3459

ISSN : 2221-4038

\section{Éditeur}

Société Internationale pour l'Histoire du Français Langue Étrangère ou Seconde

\section{Édition imprimée}

Date de publication : 1 décembre 2012

Pagination : 129-149

ISSN : 0992-7654

\section{Référence électronique}

Laurent Puren, "L'instruction en français du Chinois Kan Gao sous la Restauration à Paris »,

Documents pour l'histoire du français langue étrangère ou seconde [En ligne], 49 | 2012, mis en ligne le 04 juillet 2016, consulté le 28 mai 2021. URL : http://journals.openedition.org/dhfles/3459 ; DOI : https:// doi.org/10.4000/dhfles.3459

Ce document a été généré automatiquement le 28 mai 2021.

\section{(C) SIHFLES}




\title{
L'instruction en français du Chinois Kan Gao sous la Restauration à Paris $^{1}$
}

\author{
Laurent Puren
}

1 Parmi la galerie de portraits conservée au musée national du Château de Versailles, il en est un qui retiendra particulièrement notre attention ici. Connu sous deux appellations différentes - «Un Chinois prenant le frais sur une terrasse au bord de la mer» et «Kan Gao, Chinois de Cayenne»-, le tableau est l'œuvre de Pierre-Louis Delaval, l'un des plus célèbres portraitistes de la première moitié du XIXe siècle. Tout dans ce portrait de $1821^{2}$ témoigne de l'influence de l'exotisme dans les arts et lettres tel qu'il commençait à se manifester sous le régime de la Restauration: la technique utilisée (la peinture est réalisée intégralement avec des pigments ramenés de Chine), le personnage, aux traits asiatiques, ses vêtements, ses différents accessoires, enfin, le paysage maritime en arrière-plan. Si les deux intitulés de ce portrait convergent pour nous indiquer que le personnage est chinois, le second nous apporte des précisions sur son identité ( « Kan Gao ») et sur ce qui pourrait être interprété comme le lieu dont il est originaire ("Cayenne»), voire le décor dans lequel l'artiste lui a fait prendre la pose. Or, nous le verrons, si à l'époque où ce tableau a été réalisé, le dit Kan Gao devait effectivement se rendre en Guyane française, il ne foulera jamais des pieds le sol de cette colonie. Ce portrait a en fait été réalisé alors qu'il était de passage à Paris pour suivre ce qu'on appellerait aujourd'hui des "cours intensifs de français" sous la conduite d'une "Mme Celliez », auteur en 1821 et 1822 de deux rapports adressés au Ministre de la Marine dans lesquels elle présente les différents procédés pédagogiques auxquels elle a eu recours ainsi que les difficultés et progrès de son élève. Après avoir retracé les circonstances de l'arrivée de Kan Gao à Paris en ce début de XIX ${ }^{\mathrm{e}}$ siècle, nous nous intéresserons aux questions didactiques soulevées par la pédagogie mise en œuvre par Celliez. 


\section{L' « Expédition d'Asie » : recrutement de Chinois des Philippines à destination de la colonie guyanaise}

2 Nous sommes le 1er janvier 1819, il est 8 heures du matin : deux navires, la flûte Le Rhône et la gabarre La Durance, quittent la rade de l'île d'Aix, au large de Rochefort, pour mettre le cap sur la Guyane qu'ils atteindront après vingt-six jours de mer. L'expédition maritime, dite "Expédition d'Asie », placée sous le commandement du capitaine de vaisseau Pierre-Henri Philibert ${ }^{3}$, prend place dans les voyages d'exploration qui se multiplient à partir du milieu du XVIII siècle.

3 L'un des objectifs de cette mission est de nature scientifique, et plus précisément botanique. Georges Samuel Perrottet, embarqué en qualité de «botaniste-agriculteur du Gouvernement ", a pour tâche de collecter des plantes tropicales dans les différentes escales ponctuant leur long périple à travers les mers du Sud pour tenter ensuite de les acclimater et de les naturaliser dans les colonies françaises, notamment sur l'île Bourbon et en Guyane. Mais l'objectif est également clairement politique : il s'agit de servir les intérêts coloniaux de la France en prospectant de nouveaux territoires pouvant lui servir de "réservoir de main-d'œuvre coloniale» (Faivre 1954: 282) : «l'essentiel était d'organiser, pour l'avenir, un courant régulier de migration d'Indonésie vers nos colonies d'Amérique que la suppression de la traite privait de l'afflux des Noirs » (Ibid.). En l'occurrence, la mission s'inscrivait dans l'entreprise de colonisation agricole de la Guyane qui, depuis le XVIII siècle, donnait du fil à retordre aux gouverneurs qui se succédèrent à la tête de cette colonie, tant les conditions de vie y étaient hostiles. Philibert, que l'historien Jean-Paul Faivre désigne comme l'homme à l'origine de cette expédition d'Asie, était parvenu, dès la fin de l'été 1817, à convaincre les plus hautes autorités françaises de l'intérêt de son projet consistant à importer dans la colonie guyanaise 200 travailleurs chinois recrutés à Java pour y introduire la culture du thé sur la rive gauche de la rivière Kaw (commune de Régina).

La Guyane ne constituait donc qu'une étape, la première d'un long périple que devait entreprendre la flotte. L'escale, qui visait essentiellement à préparer l'arrivée de la main-d'œuvre, fut l'occasion de débarquer différents objets, vivres et instruments aratoires et de mettre à la disposition du gouverneur de la colonie un ingénieur des ponts et chaussées chargé de la construction d'habitations destinées à héberger les Chinois. Le Rhône et La Durance appareillèrent de l'île du Père, au large des côtes guyanaises, le 27 février pour cingler vers le Cap Vert, qu'ils atteignirent le 10 avril, puis, de là, vers l'île Bourbon, où passagers et membres d'équipage débarquèrent le 26 juin. L'escale d'un mois sur l'île Bourbon fut l'occasion de descendre à terre plusieurs caisses de végétaux collectés par Perrottet en Guyane. C'est ainsi que la vanille y fut introduite pour la première fois. La division leva à nouveau l'ancre le 27 juillet pour faire voile vers Surabaya, à l'est de Java, destination qu'elle atteignit le 13 septembre. Il s'agissait désormais pour le capitaine Philibert de passer à la phase cruciale de recrutement de la main-d'œuvre agricole.

5 Les choses ne se passèrent toutefois pas comme prévu, Philibert se heurtant rapidement au refus des autorités coloniales hollandaises de laisser embarquer le moindre homme à bord. «N'ayant pu remplir à Surabaya l'objet principal de ma mission ", écrit-il dans un rapport le 20 septembre 1820, "je vis qu'il fallait aller à Manille » (cité par Faivre $1954: 282$ ). Le voyage jusqu'à Manille nécessita plus de 2 mois supplémentaires (du 15 octobre au 22 décembre) mais ce fut la même déconvenue qui 
attendait le capitaine sur place, le gouverneur n'accédant qu'avec les plus grandes réticences à sa demande. Il réussit néanmoins dans les semaines qui suivirent à recruter, par l'intermédiaire d'un capitaine chinois influent qui lui avait été recommandé par le gouverneur, mais dut revoir sérieusement à la baisse ses ambitions, tant quantitativement que qualitativement. Philibert, décidément en proie à toutes les hostilités, dut en effet faire face à la malveillance d'une partie de ses officiers. Décidés à saborder le recrutement, ceux-ci persuadèrent le capitaine chinois que ses hommes seraient réduits en esclavage sitôt débarqués à Cayenne (Perrottet 1831 : 522-523; De St Amant 1822 : 128-129). Les recrues, conservant les arrhes et les cadeaux qui leur avaient été donnés, refusèrent d'embarquer. Afin de contrecarrer le pouvoir de nuisance des mutins, Philibert consigna tout l'équipage à bord. Finalement, sur les 200 Chinois escomptés, il n'en convainquit que vingt-sept à signer un contrat pour la Guyane, et dut se résoudre à accepter le tout venant, si on en croit Pierre-Charles de Saint-Amant ${ }^{4}$, ce qui n'augurait rien de bon pour la future réussite de l'entreprise :

Mais sur ces 27 Chinois, il ne se trouvait que deux cultivateurs : les autres étaient cuisiniers, savetiers, vagabonds et paresseux, qui vivaient dans la crapule et dans la misère, et qui ne pouvaient rien perdre à changer de pays. M. Philibert avait été obligé de prendre tout ce qui se présentait. Il fut encore trop heureux d'en pouvoir exporter quelques-uns (De Saint-Amant $1822: 134$ ).

6 A ces vingt-sept Chinois, vinrent s'ajouter cinq Indiens (ces derniers n'étant apparemment pas voués aux mêmes fonctions) et un autre Chinois - et c'est ici que nous retrouvons Kan Gao - pressenti pour diriger la future plantation de thé. De Kan Gao, nous savons qu'il était le fils d'un marchand chinois installé dans la ville d'Émouy, encore orthographié Émoui, ou Amoy, située sur les côtes sud-est de la Chine, en face de l'actuelle île de Taïwan. Ce port, aujourd'hui connu sous le nom de Xiamen, connaissait alors une intense activité commerciale, notamment avec les Philippines où était implantée une forte communauté chinoise œuvrant dans l'import-export de marchandises. Parmi celle-ci se trouvait un des oncles de Kan Gao, Kansoui, que son neveu décrit comme le "chef de la communauté chinoise établie à Manille " (Celliez 1822 : 16). Alors qu'il est âgé de 15 ans, Kan Gao est envoyé par son père chez cet oncle auprès duquel il passera les douze années suivantes et dont il deviendra le secrétaire. La rencontre de Philibert et de Kan Gao a lieu en janvier 1820 ; le capitaine parvient à le convaincre de s'exiler à Cayenne "pour y occuper ", écrira plus tard Kan Gao, "une place analogue à celle de mon oncle à Manille " (Ibid.). L'accord se concrétise sous la forme d'un engagement, «écrit en chinois, espagnol et français », stipulant les termes du contrat, et signé par Kan Gao, Philibert, Kansoui ainsi qu'" un autre capitaine français » (probablement le capitaine de frégate Georgette-Dubuisson, commandant La Durance, vaisseau à bord duquel Kan Gao devait être transporté). Nous n'avons pas pu consulter ce document, mais l'engagement devait vraisemblablement porter sur l'attribution de terres en Guyane (" on m'a promis des terres en toute propriété ») et sur un séjour préalable en France, à la charge des autorités de ce pays, pour lui permettre d'apprendre le français («mes relations devant être avec le gouvernement français, je devais savoir le français comme mon oncle sait l'espagnol ») (Ibid. : 16-17).

7 Le Rhône et La Durance mettent sous voile le 17 mars 1820 et atteignent Bourbon le 5 mai. Le mois suivant, les deux navires appareillent chacun pour des destinations différentes. Le 1er juin, Le Rhône, avec à son bord la main-d'œuvre chinoise et Perrottet, fait voile vers Madagascar qu'il atteint cinq jours plus tard puis, de là, vers la Guyane au large de laquelle la flûte mouille le 10 août. Le 13 juin, c'est au tour de La Durance de 
prendre la mer pour le voyage du retour. Après une circumnavigation de vingt-et-un mois, le 18 septembre 1820, la gabarre jette enfin l'ancre dans la rade de l'île d'Aix. Philibert et son protégé Kan Gao débarquent dans le port de Rochefort, avant de gagner Paris.

\section{La difficile instruction en français de Kan Gao}

\subsection{La prise en main de Kan Gao par Mme Celliez}

8 À Paris, Kan Gao s'installe, semble-t-il, chez le capitaine Philibert. Un mois après leur retour en France, en octobre 1820, celui-ci prend contact avec Mme Celliez à qui il confie l'instruction en français de son protégé. Adelaïde Celliez, née comtesse De Rossi, est l'épouse d'un médecin. Elle est âgée de 42 ans au moment où elle entreprend l'instruction de Kan Gao. Devenue institutrice après la Révolution, elle se consacre dès lors à l'instruction des "jeunes personnes du sexe", ainsi que les jeunes filles étaient parfois qualifiées à l'époque, et acquiert une petite notoriété dans le domaine. Dans son ouvrage de 1812 sur l'Esprit de la méthode d'éducation de Pestalozzi, Marc-Antoine Jullien (fils), révolutionnaire, homme de lettres et pédagogue, consacre une note de bas de page à cette "dame française d'un rare mérite », louant la méthode que celle-ci met en œuvre pour enseigner la géographie. En 1817, Celliez publie un Traité d'enseignement et d'éducation qui lui vaut quelques pages de recension dans l'édition d'octobre de la même année du Journal d'Éducation.

9 Les journées de Kan Gao sont désormais rythmées par les études qu'il consacre au français: le matin, l'homme, décrit par un journaliste comme "naturellement studieux ", fréquente des bibliothèques où on le voit prendre beaucoup de notes (Extrait du journal Le Figaro du 20 mai 1829, cité par Paulmier $1844: 55$ ), en soirée, il se rend au 6 rue Favart, pour suivre ses cours particuliers au domicile de son éducatrice, comme il l'explique lui-même :

Je viens chez madame Celliez tant que je veux et elle me donne de 2 à 3 heures de leçons ; pendant tout le premier hiver que j'ai été à Paris je restais chez elle tous les jours depuis 6 heures du soir jusqu'à 10 ou 11, que le domestique (sic) de M. Philibert venait me chercher (Celliez $1822: 18$ ).

Celliez met à contribution toute la famille, et en particulier ses enfants -ses deux filles de 18 et 12 ans et son fils de 15 ans -, pour la seconder dans l'entreprise de francisation du Chinois qui lui a été confiée par le capitaine Philibert. Elle introduit également son élève dans "sa société ", en le présentant, comme l'expliquera plus tard Kan Gao luimême, " chez beaucoup de ses connoissances (sic) ", notamment chez les Langlois " qui ont eu aussi toutes sortes d'attentions pour moi » (Ibid.). Le père, artiste-peintre - il s'agit peut-être de Claude Louis Langlois, dit "Langlois de Sézanne »-, auprès duquel il est possible qu'il ait suivi des cours de dessin, en profitera pour réaliser un portrait lithographié de Kan Gao. Ajoutons, pour clore cette partie sur la vie sociale et mondaine du jeune Chinois, que celui-ci a été présenté au roi Louis XVIII le 8 octobre 1821.

Nous avons un aperçu assez précis des procédés pédagogiques mis en œuvre par l'éducatrice grâce à deux documents qui ont traversé le temps jusqu'à nous. Le premier est le rapport, conservé aux Archives Nationales d'Outre-Mer, que Celliez adresse le 26 juillet 1821 au baron Portal d'Albarèdes, alors Ministre secrétaire d'état de la Marine et des colonies ${ }^{5}$. Le second document, dans lequel on trouve une version modifiée de ce 
premier rapport, contient également un second rapport, beaucoup plus succinct, adressé en février 1822 au marquis de Clermont Tonnerre, qui avait pris la succession de Portal au portefeuille du Ministère de la Marine, ainsi que diverses considérations de Celliez et de Kan-Gao ${ }^{6}$ que nous avons déjà évoquées ou évoquerons plus en avant. L'ouvrage paraît en 1822 sous l'intitulé: Historique de l'instruction du Chinois qui a été présenté au Roi, le 8 octobre 1821, par M. Philibert, capitaine de vaisseau.

Dans son rapport de 1821, Celliez commence par souligner la difficulté de sa tâche :

Il faut observer que je n'avais aucun moyen de me faire entendre de mon élève

[...] Je n'avais aucun moyen de comparaison entre deux langues dont le génie est absolument différent, dont les signes n'ont aucune similitude, et qui n'offrent pas le

plus léger point de contact entre elles.

13 Kan Gao n'a, de toute évidence, reçu aucune instruction en français préalable à son embarquement. A-t-il mis à profit le long périple maritime qui l'a conduit jusqu'aux côtes françaises pour apprendre "sur le tas ", au contact de l'équipage, quelques bribes de cette langue? Sans doute, mais son niveau devait probablement être proche d'un niveau A.0 "en route vers le A1.1", pour reprendre l'échelle et la terminologie du CECRL. "Pour être en état de nous communiquer réciproquement quelques idées ", constate la pédagogue, «il fallait parvenir à donner à M. Kan-gao (sic) une partie de l'usage qu'a ordinairement un enfant de six ans » (Celliez $1822: 9$ ).

\subsection{Les difficultés de Kan Gao en français}

Les difficultés de Kan Gao, telles que décrites par l'éducatrice, sont d'abord de nature phonétique. Certains phonèmes lui sont étrangers, c'est le cas de la « touche labiale $V$, la roulante $R$, les voix $u$, ou et quelques nazales (sic)». La réalisation de la liquide [R], ou plutôt l'impossibilité devant laquelle se trouve Kan Gao de réaliser ce phonème, provoque apparemment chez lui un effet désopilant : «mon Chinois [...] riait aux éclats quand on prononçait cette touche devant lui » (Celliez 1822: 6). "J'ai cru remarquer [...] que les sons ne sont pas distincts chez eux, comme dans les langues d'Europe ", note encore Celliez, «d'où il résulte pour un Chinois une difficulté organique pour prononcer distinctement». Les réalisations postérieures et fermées qui semblent caractériser la langue maternelle de Kan Gao («la plupart des mots chinois sont terminés par une touche fermée, ou de la gorge»), s'opposent aux réalisations françaises, antérieures et ouvertes, ce qui explique les difficultés de l'apprenant chinois à maîtriser la phonologie du français. Les confusions de Kan Gao sont fréquentes : « il confond encore le $l$ et le $d$, l'é et l'i, ou avec au, an avec on, un avec in, $u$ avec ou et ne comprend jamais eu ».

15 Celliez remarque également les différences qui séparent les deux langues en termes de réalisation prosodique : "Il s'imagine que notre langue est monotone, et en effet elle l'est par rapport à la sienne qui a quelque chose de chanté qui n'est pas sans harmonie ». Kan Gao ne parvient pas à trouver le bon «ton", la bonne «cadence » quand il lit en français.

16 Face à l'ensemble de ces obstacles, la pédagogue multiplie les approches: "Aucun moyen d'arriver au but n'est négligé : le faire écrire sous la dictée, le faire copier, le faire lire, le faire épeler, lui prononcer vingt fois la même chose, la lui faire répéter, lui démontrer les mouvements de l'instrument vocal, rien n'est épargné ». 
17 Kan Gao, locuteur d'une langue isolante/analytique éprouve également des difficultés à maîtriser le système morphologique du français, et notamment le système de flexion caractéristique des langues dites justement "flexionnelles». Celliez ne le pose évidemment pas en ces termes mais affine, dans la version remaniée de son premier rapport, le jugement péremptoire qu'elle porte sur la langue « chinoise ».

Partant du constat suivant :

Rond et ronde lui paraissent deux mots; il rit encore quand on lui dit un cheval et des chevaux, etc. Ce fut bien pis quand il a été question des verbes. Comment faire comprendre que : avoir, j'ai, nous avons, ils avaient, nous aurons, j'aurais, etc. ne sont que des modifications de la même idée?

19 Elle conclut dans son rapport de juillet 1821: «Il n'y a [...] point de grammaire en Chine », pour tempérer un an plus tard :

J'ai lieu de supposer qu'il n'y a point de grammaire chinoise ou au moins qu'elle n'a point d'analogie avec les grammaires d'Europe, car les différences orthographiques de genre, de nombre, et celles de la conjugaison m'ont paru des idées qui étaient absolument étrangères à mon élève (Celliez 1822 : 7).

\subsection{Une pédagogie traditionnelle en phase avec son époque}

20 Les procédés pédagogiques utilisés par l'éducatrice, tels que nous allons maintenant les présenter, doivent, pour être bien compris aujourd'hui, être replacés dans leur contexte historico-éducatif.

L'enseignement des LVE (langues vivantes étrangères) sous la Restauration n'est pas encore entré dans une phase d'institutionnalisation. Paul Lévy note à ce sujet: «la réforme des lycées sous Napoléon et en particulier presque tous les règlements qui se suivent de 1800 à 1830, ignorent les LV » (cité par Puren 1988: 45). Il n'existe pas de critères précis liés au recrutement des enseignants de LVE dont la matière n'est proposée dans les collèges royaux qu'à titre facultatif, à l'instar de la musique ou de la danse, «pendant les heures de récréation » (Rothmund 2005). Il faudra attendre 1838 pour que cet enseignement devienne obligatoire, et 1840 pour qu'une circulaire définisse et impose pour la première fois une méthodologie officielle d'enseignement/ apprentissage des LVE, connue aujourd'hui sous l'appellation de "grammairetraduction ». Comme son nom l'indique, celle-ci, alignée sur les procédés en cours dans l'enseignement des langues mortes, se caractérise par la prééminence accordée à un enseignement explicite et déductif de la grammaire et par la place centrale occupée par le thème et la version. On est face à un enseignement décontextualisé, axé sur l'écrit, l'activité de l'apprenant, traduction mise à part, se réduisant pour l'essentiel à la mémorisation et la restitution de règles, de conjugaisons ou encore de listes de vocabulaire. Même si ce courant méthodologique n'a pas encore été officialisé dans les années 1820 , les différents procédés le composant étaient déjà probablement en partie appliqués dans les classes du secondaire, pour les élèves qui choisissaient d'étudier une LVE.

Les convergences méthodologiques entre l'enseignement des LVE sous la Monarchie de Juillet et celui du FLM (français langue maternelle) sous la Restauration sont évidentes : la grammaire, dans l'un et l'autre cas, y occupe une place centrale, de même que les procédés décrits précédemment (mémorisation, récitation). Nouvelle discipline instituée à partir de la seconde moitié du XVIII ${ }^{e}$ siècle dans l'enseignement secondaire, «la grammaire française », souligne André Chervel, «se récite exactement comme la 
grammaire latine ou les fables de la Fontaine» (Chervel 2006: 241). L'analyse grammaticale (repérage des "parties du discours») et l'analyse logique (découpage de la phrase en sujet, copule et attribut et identification des propositions) sont au cœur de la première grammaire scolaire qui occupe les devants de la scène jusqu'au milieu du XIX ${ }^{e}$ siècle. L'orthographe n'est pas oubliée: une partie non négligeable du volume horaire des établissements secondaires est dévolue aux dictées et cacographies (textes fautifs que l'élève doit corriger).

Logiquement, on retrouve dans les procédés pédagogiques utilisés par Celliez la prééminence accordée à la langue écrite, à travers la place prépondérante qu'occupent la grammaire (conjugaisons, analyses grammaticales, analyses logiques) et l'orthographe (dictées, cacographies). Le recours à la traduction est également présent dans deux types d'exercices. L'éducatrice fait remplir à Kan Gao un tableau à trois colonnes, avec d'un côté, en français, des "petites propositions et phrases familières " et, de l'autre, en caractères "chinois", leur traduction et une transcription de la prononciation française. Elle lui fait également tenir un "syllabaire comparé», dans lequel, explique la pédagogue, Kan Gao «a figuré, autant qu'il l'a pu, en caractères chinois, toutes nos voix et toutes nos syllabes".

Cette place accordée à la langue de son élève mérite deux remarques. En premier lieu, la transcription, forcément approximative, des phonèmes du français en caractères chinois à laquelle se plie Kan Gao, tout au moins au début de son apprentissage, illustre l'importance donnée par Celliez à la prononciation, qui reste néanmoins fortement corrélée à l'écrit, puisque la maîtrise de celle-ci détermine la capacité de lecture d'un texte et, inversement, de restitution d'un écrit oralisé à travers l'exercice fétiche de l'école française qu'est la dictée, auquel est régulièrement confronté son élève. L'approche adoptée ici rappelle celle préconisée par les instructions officielles de 1840 consacrant la méthode grammaire-traduction, dans laquelle la prononciation était mise au service de l'écrit («accoutumer [...] l'oreille par des dictées fréquentes, [faire] apprendre par cœur et réciter convenablement les morceaux dictés ») plus qu'à celui du développement d'une compétence de communication orale. À l'exercice de version, Celliez ajoute un exercice de thème pour lequel elle fait appel à la participation de l'une de ses filles :

[...] j'ai feint que ma fille voulait apprendre le chinois alors nous faisons la contre partie ; il nous dit le mot, nous le figurons en caractères français. Le lendemain, ma fille prononce le chinois et nous tâchons que M. Kangao dise le français (Celliez $1822: 17)$.

La deuxième remarque est liée à l'utilisation par l'éducatrice de ce terme générique, le "chinois», qui donne l'illusion que la Chine serait caractérisée par une situation sociolinguistique homogène et monolithique : tout Chinois ne parlant nécessairement que «le » chinois. La réalité est évidemment plus complexe. Le sinologue Jean-Pierre Abel-Rémusat, dans le compte rendu très sévère ${ }^{7}$ qu'il établit de l'ouvrage de Celliez de 1822, note au sujet de Kan Gao :

Quoiqu'il eût étudié, et qu'il sût même écrire, il ne parlait pas la langue mandarinique, et il ne savait que le patois de son pays. Il avait autrefois appris par cœur les livres de Confucius, et toutefois il ne savait pas faire usage du dictionnaire chinois de l'empereur Khang-hi (Abel-Rémusat 1829 : 262). comme langue écrite officielle, celle probablement dans laquelle était rédigé le 
dictionnaire unilingue qu'elle s'était procuré, et la variation dialectale que parle son élève :

Nous n'avons point de dictionnaire français et chinois. Je n'ai pu retirer aucun avantage du dictionnaire chinois, parce qu'il est dans la langue de Canton ${ }^{8}$ et que $\mathrm{M}$. Kangao parle celle d'Émoui, de plus M. Kangao a quelques préventions particulières ; il soutient que le dictionnaire n'est pas exact [...]

Cette langue écrite, normalisée, constituait par conséquent une langue que l'on pourrait qualifier de «seconde » pour Kan Gao, ce d'autant plus que dans son exil aux Philippines, il avait probablement connu une forme d'acculturation linguistique à travers une confrontation à de nouvelles variétés langagières : le "chinois " tel que pratiqué par la diaspora, les langues locales, l'espagnol également, comme langue du colonisateur. On peut, par conséquent, se demander si, en faisant de ce dictionnaire un support central de son instruction, Celliez n'ajoutait pas une difficulté à une autre difficulté. Elle note ainsi : «il prétend que de parler même en chinois le fatigue, et on peut s'apercevoir quand il lit le chinois qu'il est en effet promptement fatigué ».

\subsection{Intuition sensible et pratiques innovantes}

Si le cœur de la méthodologie mise en œuvre par l'éducatrice relevait, nous l'avons vu, d'une approche traditionnelle, axée sur un apprentissage formel de la langue écrite, on trouve également dans les approches pédagogiques évoquées dans ses rapports, des éléments novateurs préfigurant la méthode directe et plus généralement les méthodes actives tournées vers une vision pratique de l'enseignement/apprentissage et privilégiant la dimension orale de la langue cible.

Kan Gao apprenant le français en situation d'immersion, en milieu homoglotte, cette langue est celle de l'environnement dans lequel il évolue et est amené à interagir au quotidien. Son instruction vise par conséquent un objectif pratique, même si celui-ci, nous l'avons $\mathrm{vu}$, n'apparaît pas nécessairement comme prioritaire au vu de l'importance accordée à un enseignement formel de l'écrit. Dans les sorties mondaines, et peut-être culturelles, que lui organise l'éducatrice, dans ses contacts quotidiens avec les enfants de celle-ci, Kan Gao est amené à pratiquer le français en situation réelle avec des locuteurs natifs. Celliez dit ainsi employer de " petits moyens pour le faire parler, et pour lui donner des leçons indirectes, qui lui sont souvent aussi utiles que les leçons directes». Cet enseignement "indirect» passe notamment par le jeu. "Madame Celliez», écrit ainsi Kan Gao, "m'a montré plusieurs jeux de cartes afin que je m'ennuyasse un peu moins, et afin que je fusse obligé de prononcer quelques mots français » (Celliez 1822: 17). Pour l'enseignement lexical, qui pose des problèmes à Kan Gao ("S'il a la mémoire des choses ", note Celliez, "il n'a pas celle des mots»), l'éducatrice indique se servir « des moyens employés avec les sourds-muets »:

présenter l'objet, en écrire le nom, le prononcer puisque j'avais à faire à un parlant, le faire réciter, nommer l'objet et le lui faire montrer, ajouter les qualités de l'objet, appliquer ces même qualités à un autre objet, composer de suite une proposition avec les noms, les qualités et les verbes connus, etc.

Si on fait abstraction de la phase écrite mentionnée par la pédagogue, la méthode que celle-ci décrit ressemble en tous points à l'approche dite de l'« intuition sensible " ("Anschauung ») mise en œuvre dès la fin du XVIII ${ }^{e}$ siècle par Pestalozzi dans ses orphelinats suisses. Les principes la caractérisant - priorité accordée à l'observation d'objets réels ou d'images les représentant, à la manipulation, à l'action, à l'oral - sont 
bien connus des réformateurs de l'éducation qui, depuis le XVI siècle, de Montaigne à Rousseau, en passant par Rabelais et Comenius, vantent les mérites de la "méthode naturelle ». Nous avons montré ailleurs (Puren 2004) que la réforme éducative majeure accomplie en Allemagne au début du XIX ${ }^{e}$ siècle s'est faite en grande partie sur la base de ce principe éducatif qui a ensuite transité par l'Alsace, avant d'être finalement adopté par les éducateurs français sous la IIIe République. Incarnée notamment par les "exercices d'intelligence et de langage», connus également sous l'expression d'« exercices de pensée et de parole ", l'approche intuitive est à l'origine de la méthode dite " maternelle » préconisée par Irénée Carré pour franciser d'abord les Bretons puis l'ensemble des élèves alloglottes de France et des colonies (Puren 2003), celle-là même qui, en 1902, sous l'appellation de "méthode directe», deviendra la méthodologie officielle d'enseignement/apprentissage des LVE. Il est fort à parier que Celliez connaissait Pestalozzi qui était son contemporain. Comme souligné plus haut, l'éducatrice, pour son approche jugée novatrice de l'enseignement de la géographie, est citée par Marc-Antoine Jullien dans son ouvrage de 1812 consacré à Pestalozzi. Or, cet auteur connaissait très bien le pédagogue suisse qu'il avait rencontré à Yverdon, avec lequel il avait ensuite entrepris une correspondance et auquel il avait confié l'éducation de ses trois premiers fils.

La mention effectuée plus haut par Celliez aux méthodes utilisées avec les sourds-muets mérite ici un petit développement. L'éducatrice avait fait appel à l'abbé Paulmier en sa qualité d'instituteur des sourds-muets de l'Institution royale de Paris. Élève et collaborateur de l'abbé Sicard, le fondateur de l'Institution, qui avait lui-même pris la succession du célèbre abbé de l'Épée, pionnier dans l'enseignement spécialisé des sourds-muets, Paulmier s'était proposé d'entrer en communication avec Kan Gao à travers le "langage d'action", c'est-à-dire en recourant essentiellement à la pantomime.

$\mathrm{Au}$ XVIII ${ }^{\mathrm{e}}$ siècle, les cercles intellectuels français, notamment des philosophes comme Rousseau et Condillac, débattent avec passion de la genèse et de l'universalité du langage. Dans la perspective évolutionniste qui les animent, le langage d'action ou gestuel est perçu comme le «langage naturel du genre humain » qui aurait précédé la langue parlée, fruit d'une convention. On croit alors à la dimension univoque du lien entre geste et pensée qui s'opposerait à celle équivoque de la relation entre mots et pensée (Rosenfeld 2001 : 92). De ce point de vue, le sauvage comme le sourd, considérés comme des êtres non civilisés, encore "à l'état de nature ", incarnent "l'homme d'avant le langage » avec lequel il est possible d'instaurer un dialogue au moyen de la pantomime (Branca-Rosoff 2008 : 91-92). Ce n'est pas un hasard si Victor de l'Aveyron, à l'initiative de l'abbé Sicard, est confié à Itard, nommé médecin chef de l'Institution accueillant les jeunes sourds. L'abbé Sicard avait lui-même, à l'occasion d'un exercice public de rééducation comme il en faisait souvent, mené une expérience avec TchongA-Sam, un Chinois arrivé en France en 1800, dans le double objectif « de démontrer la nature du langage des signes et de donner à l'audience une idée de la langue chinoise " (Rosenfeld 2001 : 340). Plus de vingt ans après son maître, l'abbé Paulmier intervient donc lui aussi, à la demande de Celliez, auprès d'un Chinois non francophone avec lequel il essaie d'entrer en communication par le biais de la pantomime, véritable " sémiotique visuelle" (Branca-Rosoff 2008: 95) inventée par Sicard en 1808 dans sa Théorie des signes et matérialisée par une succession de saynètes. Si pour certains termes, en particulier ceux représentant une action concrète à dimension universelle, 
la pantomime pouvait conduire à une interprétation univoque, il n'en était pas de même pour des termes renvoyant à des concepts abstraits. Ainsi des verbes « naître " et «abjurer » tels que présentés dans le dictionnaire de Sicard :

NAÎTRE. $1^{\circ}$ Supposer et figurer une femme grosse. $2^{\circ}$ La représenter, par signes, dans les douleurs de l'enfantement, et donnant le jour et la vie à un enfant. $3^{\circ}$ Mode indéfini.

ABJURER. $1^{\circ}$ Représenter un homme qui étoit né dans une fausse religion, et qui professoit une mauvaise doctrine; ces signes se font en figurant une religion que Dieu n'a pas révélée, ou qui a été défigurée et altérée par les hommes. $2^{\circ}$ Action de déclarer publiquement qu'on s'est trompé, qu'on abandonne cette religion, et qu'on embrasse la véritable. $3^{\circ}$ Mode indéfini (Cité par Branca-Rosoff 2008 : 95).

Dans ce jeu de mimes, cette partie de Pictionnary avant l'heure à laquelle se prêtèrent Kan Gao et ses instructeurs, on imagine aisément la perplexité de l'élève face aux mises en scène gesticulatoires auxquelles il était soumis. Celliez note ainsi: «Lorsqu'on employait la pantomime profondément métaphysique, en usage entre les sourds-muets et leurs professeurs, il n'y voyait guère qu'une chose amusante dont il ne devinait pas le but ». Le procédé, quand il ne l'amuse pas, semble l'ennuyer: «Il est tel jour où je n'obtiendrai pas qu'il tourne la tête pour regarder [...] une pantomime que je lui fais exécuter par mon fils, pour lui enseigner soit le mot fuir, soit le mot prendre, soit le mot suivre, soit tout autre ».

Kan Gao est de toute évidence peu enclin à s'approprier ce langage d'action. " Notre Chinois ", écrit Paulmier, "selon la louable coutume de ses compatriotes, n'était pas grand gesticulateur » (Paulmier $1828: 446)$.

\subsection{Des résultats incertains}

Intéressons-nous à présent aux résultats de cette instruction tels qu'ils apparaissent dans les rapports de Celliez. En juillet 1821, soit après neuf mois de cours, l'éducatrice note :

Je suis arrivée, par degrés, à mettre mon élève en état de lire le français, de comprendre une foule de choses, de conjuguer sur un modèle un verbe régulier de première et de seconde conjugaison, il n'entend pas encore bien ceux de troisième ; d'employer assez à propos le présent indicatif, le passé indéfini et le futur des verbes qui lui sont familiers [...] il commence à faire des fautes d'orthographe qui prouvent qu'il entend le système des consonances. Par exemple il écrivait hier cinq, sinque ; parce que, parsque ; pense, pance ; amitiés, amitier ; toutes choses que je rectifie à l'instant, tout en lui disant que cela est bien pour l'oreille.

Elle ajoute qu'«il est impossible d'assigner l'époque à laquelle M. Kangao sera en état de tenir une correspondance. Il comprend, il parlera avant de composer. Ce que nous avons fait était bien difficile; ce qui nous reste à faire l'est plus encore ».

Celliez, on le voit, met l'accent sur les compétences purement linguistiques de maitrise $\mathrm{du}$ code, les compétences de communication passant au second plan: Kan Gao commence à savoir sa grammaire, son orthographe et sa conjugaison, peut déchiffrer, comprendre un peu, mais en production, tant à l'oral qu'à l'écrit, les progrès semblent nettement plus incertains. L'éducatrice pointe du doigt les traits de personnalité de son élève comme autant d'obstacles au bon déroulement de l'instruction. Elle évoque ainsi le «caractère particulier de l'individu, ou plutôt de sa nation; ses entêtements, ses ennuis, son indolence commune à tous ceux qui sont nés sous un climat chaud, [qui] sont souvent les obstacles les plus difficiles à surmonter ». 
Et Celliez de souligner encore sa timidité, sa "paresse d'esprit ", son " caractère d'enfant ", "son organisation [qui] se refuse à un travail suivi », la fatigue qu'engendre chez lui « l'application », sa passivité («il ne veut essayer que ce qu'il est sûr de bien faire ", « il questionne peu »), tout en mettant en avant son " intelligence », sa " finesse d'esprit » et son caractère qui peut également se révéler enjoué : « quand il n'est pas souffrant, il est gai et malin, il se plaît à nous faire des espiègleries auxquelles nous nous prêtons ». Nous laissons de côté les jugements de valeur et autres considérations paternalistes de l'éducatrice pour ne retenir que les moments d'abattement, d'ennui, de lassitude de Kan Gao qui se comprennent aisément au vu de sa position d'exilé, totalement coupé de sa famille, et confronté à un enseignement essentiellement livresque et formel d'une langue inconnue. "Je ne sais pas encore la langue française ", notera Kan Gao en août 1822, après deux années d'instruction, " parce que c'est bien plus difficile que je ne croyais » (Celliez 1822: 17). Sept mois se sont écoulés depuis les premières remarques de Celliez sur les progrès de son élève, lorsqu'en février 1822, dans son second rapport, elle note :

Il est arrivé au point de comprendre tout ce qui est du langage familier ; de se faire comprendre des gens avec lesquels il est à son aise; de pouvoir analyser grammaticalement et logiquement des phrases faciles à comprendre, de pouvoir lire un peu seul.

Elle ajoute: "Si j'étais suffisamment secondée par les circonstances accessoires, j'oserais presque affirmer qu'il ne faut pas plus de dix-huit mois, à M. Kan Gao, pour arriver au degré de perfection qu'on doit se proposer quand on fait une pareille entreprise » (Celliez 1822).

\subsection{Les rapports tendus entre Celliez et les autorités ministérielles}

Les «circonstances accessoires» auxquelles l'éducatrice fait ici allusion tiennent essentiellement à des questions pécuniaires qui deviennent une source de tension avec les autorités ministérielles dont elle dépend pour ses émoluments. Kan Gao reçoit chaque mois $500 \mathrm{~F}$ du département de la Marine, somme destinée à couvrir l'ensemble de ses frais, dont son instruction. Celliez note : « Comme il [Philibert] ne voulut prendre sur lui aucune dépense sans une autorisation spéciale du ministre et mon élève n'avait que 15 à $20 \mathrm{~F}$ par mois pour ses dépenses particulières, mes moyens ont été très bornés " (Celliez 1822: 14). La pédagogue réclame des moyens supplémentaires pour l'achat d'ouvrages ainsi que pour un projet dans lequel il est question de recruter un interprète espagnol-français pour faire accomplir à son élève " un travail d'une utilité majeure » (Celliez 1822 : 16). Elle conçoit en effet pour celui-ci « de hautes espérances », voyant en lui «un homme précieux pour la France et unique dans l'univers; puisqu'étant chinois, sachant bien sa propre langue, il saurait aussi le français et peutêtre l'espagnol! ». "C'est une entreprise absolument neuve », écrit-elle encore, " et dont les conséquences peuvent être du plus grand intérêt pour la science et pour la philosophie ». Il existe un véritable décalage entre l'enthousiasme naîf dont fait preuve Celliez dans les projets qu'elle entrevoit pour son élève et les caractéristiques de celuici ainsi que les raisons de son séjour en France. D'une part, Kan Gao n'est pas ce que l'on pourrait appeler un lettré mais un simple marchand destiné à exercer une fonction de contremaître dans une plantation de thé. Ensuite, parce qu'il a été coupé relativement jeune de ses racines chinoises dans l'exil qui l'a conduit à Manille, il n'a qu'une connaissance imparfaite des éléments linguistiques et culturels liés à son pays 
d'origine. «On suppose les Chinois qui viennent nous visiter, aussi instruits qu'ils sont en général ignorans", souligne ainsi le sinologue Abel-Rémusat (Abel-Rémusat 1829: 263), qui ajoute, persifleur et quelque peu méprisant :

Attendre des renseignements historiques ou littéraires d'un homme de cette espèce, c'est comme si à la Chine on voulait tirer quelques lumières sur l'histoire de France, ou la littérature grecque ou latine, de quelque Bas-Breton que le hasard aurait conduit à quinze ans à la Martinique ou à la Guadeloupe, pour y diriger une plantation de sucre, et qui ne saurait parler que le langage de Cornouailles (Ibid. : 262-263).

41 Les autorités ministérielles sont également loin de partager l'enthousiasme de Celliez si l'on en croit le silence qu'elles opposent aux demandes de plus en plus pressantes de l'éducatrice. Le 3 mai 1822, face à l'absence de réaction suscitée par l'envoi de son second rapport au Ministre de la Marine, le marquis de Clermont-Tonnerre, elle adresse à celui-ci une lettre courroucée :

Puisque le silence, je dirais presque dédaigneux, que Votre Excellence garde depuis plus de deux mois, me prive d'employer un moyen que je regardais comme précieux pour [mot raturé illisible] les progrès du Chinois dans l'étude de la langue française, je suis obligée de prévenir Votre Excellence que le terme auquel j'espérais voir Mr K. parler et écrire le français sera vraisemblablement fort reculé9.

Cette lettre, conservée aux Archives nationales d'outre-mer, porte l'annotation suivante, ajoutée probablement par le ministre lui-même ou par quelqu'un de son cabinet, qui en dit long sur le décalage auquel nous faisions allusion précédemment: «Depuis 2 ans et demi que Mr Kankao (sic) coûte $500 \mathrm{~F}$ par mois au Gouvernement, non seulement il devrait parler et écrire, mais il devrait savoir beaucoup d'autres choses et il y a plus qu'il ne faut pour acheter les livres dont il peut avoir besoin ».

Dix jours plus tard, elle relance le ministre en ces termes :

[...] depuis 3 mois, je sollicite une réponse de votre Excellence. J'ai eu l'honneur de lui écrire quinze fois. Sans doute que mes lettres ne lui sont jamais parvenues. Je la supplie de prendre ma demande en considération et je la prie de bien remarquer que mes intérêts n'y sont pour rien. Je suis avec respect votre très humble et très obéissante servante.

Le marquis de Clermont-Tonnerre finit enfin par lui répondre le 17 mai 1822 en lui confirmant la réception de ses courriers et en lui indiquant qu'elle sera bientôt fixée sur sa décision relative à «l'étranger ». En réalité, personne en dehors de Celliez, et peut-être de Philibert, ne souhaite voir le séjour de Kan Gao s'éterniser, pas plus les autorités que Kan Gao lui-même. L'installation des Chinois en Guyane ayant en effet tourné au fiasco ${ }^{10}$, la présence du jeune Chinois en France n'avait plus de raison d'être.

\section{3. Épilogue}

Les évènements se précipitent à compter de l'été 1822 , avec le décès prématuré d'Adelaïde Celliez à Blois le 4 août, à l'âge de 44 ans. Désormais, plus rien ne retient Kan Gao en France. Dans les quelques lignes qu'il écrit ou fait écrire dans l'ouvrage de l'éducatrice qui paraittra après la mort de celle-ci, il dit tout son désarroi, le sentiment de solitude extrême qui l'habite. "Je suis malheureux » ou « je suis malheureux ici » ", écrit-il à trois reprises. Depuis son arrivée en France deux ans plus tôt, il est sans nouvelles de ses parents et de son oncle. S'agit-il d'une volonté délibérée de Philibert de l'isoler? A moins qu'il n'agît ainsi pour le préserver. Toujours est-il que Kan Gao dit 
avoir découvert que son protecteur n'avait jamais expédié la lettre qu'il avait écrite à son oncle Kansoui. De la même manière, il découvre inopinément, alors qu'il aide Philibert à déménager, la lettre que ses compatriotes exilés en Guyane lui ont adressée quinze mois plus tôt et que le capitaine s'est bien gardé de lui remettre, sans doute pour éviter qu'il prenne connaissance du désastre. Le courrier lui apprend, écrit-il "que 10 de mes compatriotes sont morts, il en est mort sans doute plusieurs autres depuis ». Il ajoute :

En général, je n'ai que des remercimens à faire aux Français, ils m'ont bien traité, cependant, je suis malheureux, et j'aime mieux m'en aller que d'achever d'apprendre la langue française et de faire valoir les droits que j'ai à obtenir en dédommagement du tems qu'on m'a fait perdre, puisque le gouvernement ne tient pas les engagements qu'il avoit pris (sic) (Celliez $1822: 18$ ).

Kan Gao se sent floué : il n'occupera jamais ce poste de cadre qu'on lui avait fait miroiter et pour lequel il avait consenti à tant de sacrifices, parmi lesquels l'apprentissage du français n'était pas le moindre. Il se plaint en outre du manque à gagner que son exil a engendré par rapport à sa situation antérieure et de l'impossibilité qui en découle d'adresser de l'argent à son père comme il en avait l'habitude. Il exprime le vœu de rentrer à Manille, puis à Émoui pour s'expliquer auprès de son père.

Le triste sort de Kan Gao est comparable à celui d'autres Chinois "échoués ", volontairement ou pas, en France avant lui ${ }^{11}$, qui vécurent eux aussi cet exil extrême, privés de tout moyen de communiquer avec les leurs. Uniques représentants de leur nation, Kan Gao, comme ses prédécesseurs, furent condamnés à jouer les éternels "Chinois de service », voire à être exhibés comme des bêtes curieuses. L'historienne Danielle Elisseeff, auteur d'une biographie consacrée à Arcade Hoang ${ }^{12}$, un Chinois émigré en France qui devint l'interprète et le bibliothécaire orientaliste de Louis XIV, écrit à propos de celui-ci : "Arcade vécut toujours en marge de tout: coupé de sa société originelle, étranger à la nôtre, mal formé, mal instruit, essayant tragiquement de se couler dans un moule européen " (cité par Lelièvre 2004: 325). Ce constat s'applique également, nous semble-t-il, à la situation vécue par Kan Gao.

Finalement, le 19 octobre 1822 le Ministre de la Marine fait approuver par Louis XVIII le départ de Kan Gao, prévu le mois suivant à bord d'un bâtiment de commerce à partir de Bordeaux, ainsi que l'octroi d'une sorte de "prime au retour" de $600 \mathrm{~F}$. L'histoire se finit mal. Kan Gao ne reverra jamais son oncle, ni son père, en tout cas si l'on en croit le témoignage d'Abel-Rémusat selon lequel il serait mort en mer avant d'avoir atteint les Philippines. De Kan Gao, il ne reste plus, écrit-il, que « quelques livres chinois qu'il avait laissés à Paris [et qui] ont été dispersés, dépareillés et vendus à des amateurs qui en ont donné des prix exorbitants » (Abel-Rémusat : 262). 


\section{BIBLIOGRAPHIE}

ABEL-REMUSAT, Jean-Pierre (1829). « Sur les Chinois qui sont venus en France ». In Jean-Pierre Abel-Remusat. Nouveaux mélanges asiatiques, ou recueil de morceaux de critique et de mémoires relatifs aux religions, aux sciences, aux coutumes, à l'histoire et à la géographie des nations orientales. t.1. Paris : Schubart \& Heideloff, 258-265.

BRANCA-ROSOFF, Sonia (2008). « Sicard et l'instruction des sourds-muets à Paris autour de 1800 ». In Llona Pabst (Ed.). Actes du colloque international « Idéologie-Grammaire GénéraleÉcoles Centrales ", 29 mars-2 avril 2001, Château de Hohentübingen, 88-104. [En ligne] consulté le 29 septembre 2012. URL : http://www.geisteswissenschaften.fu-berlin.de/v/ grammaire_generale/Actes_du_colloque/Textes/Branca-Rosoff/Sonia_Branca-Rosoff.pdf CELLIEZ, Adélaïde (26 juillet 1821). Rapport à son Excellence le Ministre de Marine, sur les progrès dans l'étude de la langue française du Chinois amené à Paris par M. Le Capitaine Philibert vers 1820. Archives nationales d'Outre-Mer, Carton 259, Dossier 1777.

CELLIEZ, Adélaïde (1822). Historique de l'instruction du Chinois qui a été présenté au Roi, le 8 octobre 1821, par M. Philibert, capitaine de vaisseau. Blois : Aucher-Éloy.

CHERVEL, André (2006). Histoire de l'enseignement du français du XVII ${ }^{\mathrm{e}}$ au XX $\mathrm{XX}^{\mathrm{e}}$ siècle. Paris : Editions Retz.

DE ST-AMANT, Pierre-Charles (1822). Des colonies ; particulièrement de la Guyane française, en 1821. Paris : Chez Barrois/Delaunay.

FAIVRE, Jean-Paul (1954). L'expansion française dans le Pacifique de 1800 à 1842. Paris : Nouvelles éditions latines.

GAFFAREL, Paul (1908). La politique coloniale en France de 1789 à 1830. Paris : F. Alcan.

PAULMIER, Louis-Pierre (1828). « Art d'apprendre à parler aux sourds-muets ». Le Mercure de France au dix-neuvième siècle. t. 21. Paris : Au bureau du Mercure, 441-450.

PERROTTET, Georges Samuel (1831). «Souvenir d'un voyage autour du monde ». La Revue des deux Mondes. t. 1 \& 2. Seconde édition, 470-525.

PUREN, Christian (1988). Histoire des méthodologies de l'enseignement des langues. Paris : Clé International.

PUREN, Laurent (2003). « Pédagogie, idéologie et politique linguistique. L'exemple de la méthode Carré appliquée à la francisation de la Bretagne à la fin du XIX ${ }^{\mathrm{e}}$ siècle ». Glottopol, 1, janvier, 33-53. [En ligne] consulté le 19 octobre 2012. URL : http://www.univ-rouen.fr/dyalang/glottopol

PUREN, Laurent (2004). L'École française face à l'enfant alloglotte. Contribution à une étude des politiques linguistiques éducatives mises en œuvre à l'égard des minorités linguistiques scolarisées dans le système éducatif français du XIX ${ }^{\mathrm{e}}$ siècle à nos jours. Thèse de doctorat en didactique des langues et des cultures, Paris 3-Sorbonne Nouvelle (thèse non publiée).

ROSENFELD, Sophia (2001). A revolution in language. The problem of signs in late eighteenthcentury France. Stanford : Stanford University Press.

ROTHMUND, Elisabeth (2005). « Manuels, auteurs et éditeurs dans les premières décennies de l'enseignement scolaire de l'allemand ». Histoire de l'éducation, 106, 15-40. [En ligne] consulté le 02 octobre 2012. URL : http://histoire-education.revues.org/1076 


\section{NOTES}

1. Nous proposons une version intégrale de cet article, accompagnée de différents documents, dont les 2 rapports de Celliez, sur le site suivant : http://kangao1820.wordpress.com/

2. Le portrait accompagné d'une analyse historique et artistique est accessible à cette adresse : http://www.histoire-image.org/pleincadre/index.php?i=452 (page consultée le 12/07/12).

3. Créole né en 1774 à Saint-Denis, à l'île Bourbon (La Réunion), Philibert était un officier de marine aux états de service impressionnants. Nommé, en 1822, député de l'île Bourbon à Paris par le roi, il meurt en 1824 .

4. Secrétaire particulier du baron de Laussat, gouverneur de la Guyane.

5. Pour nous faciliter la tâche, les citations qui suivent ne mentionnant aucune source sont extraites de ce rapport, à la différence de celles tirées de son ouvrage de 1822 pour lesquelles la source et la pagination apparaîtront systématiquement.

6. Ces pages ont été rédigées, ou plus vraisemblablement, vu son faible niveau de français, dictées par Kan Gao le 7 août 1822, soit 3 jours après le décès de Mme Celliez.

7. «Il eût suffi peut-être d'une simple annonce pour cet opuscule: il ne se recommande à l'attention du public, ni par son étendue, ni par son contenu, ni par sa forme " (Abel Rémusat $1829: 258)$.

8. Celliez est-elle victime d'une confusion avec le mandarin lorsqu'elle évoque «la langue de Canton » à propos du dictionnaire?

9. Archives nationales d'Outre-Mer, Carton 259, Dossier 1777.

10. Voir l'article complet sur http://kangao1820.wordpress.com/

11. Voir en particulier : LELIEVRE, Dominique (2004). Voyageurs chinois à la découverte du monde. De l'Antiquité au XIX ${ }^{\mathrm{e}}$ siècle. Genève : Ed. Olizane.

12. ELISSEEFF, Danielle (1985). Moi, Arcade : interprète chinois du Roi-Soleil. Paris : Arthaud.

\section{RÉSUMÉS}

De retour de son « Expédition d'Asie » en octobre 1820, le capitaine Philibert ramène à bord de La Durance un jeune marchand chinois du nom de Kan Gao. Celui-ci, recruté à Manille 10 mois plus tôt pour diriger la plantation de thé que le Ministère de la Marine et des colonies tente de créer en Guyane française, doit préalablement à ce séjour suivre des cours de français à Paris. C'est une Madame Celliez, institutrice de son état, qui, pendant près de 2 ans, entre 1820 et 1822, se charge de l'instruction de Kan Gao. Les deux rapports qu'elle adresse au Ministre de la Marine en juillet 1821 puis en février 1822, nous renseignent sur les procédés pédagogiques utilisés par l'éducatrice.

On his return from his « Expedition in Asia » in October 1820, Captain Philibert brought aboard La Durance a Chinese man named Kan Gao. The young Asian merchant had been recruited in Manila 10 months earlier to run the tea plantation that the Ministry of The Marine and Colonies was endeavouring to set up in French Guiana. Prior to this trip, French courses were organised in Paris for Kan Gao. Mrs Celliez was the teacher to whom Kan Gao's French instruction was entrusted. The two reports she sent to the Minister of the Navy in July 1821 and February 1822, provide us with information on the teaching methods she used during this period of instruction lasting for almost 2 years. 
INDEX

Keywords : Restauration, Chinese, nautical expedition, colonisation, immigration, instruction in French, language immersion

Mots-clés : Restauration, Chinois, expédition maritime, colonisation, immigration, instruction en français, immersion linguistique

\section{AUTEUR}

\section{LAURENT PUREN}

Université de La Réunion

laurent.puren@gmail.com 